\title{
Enumeration of Kekule Structures of Polyhex Monoradical Having Only Linear Assemblies of Benzenoid Hydrocarbons
}

\author{
Alphonse Emadak*, Robert Martin Nemba, Leonel Tsassi Djoumessi, Jules Cesar Epee Ndongue
}

Department of Inorganic Chemistry, Faculty of Science, University of Yaounde I, Yaounde, Cameroon

Email address:

emadak@gmail.com (A. Emadak)

${ }^{*}$ Corresponding author

\section{To cite this article:}

Alphonse Emadak, Robert Martin Nemba, Leonel Tsassi Djoumessi, Jules Cesar Epee Ndongue. Enumeration of Kekule Structures of Polyhex Monoradical Having Only Linear Assemblies of Benzenoid Hydrocarbons. Science Journal of Chemistry.

Vol. 7, No. 3, 2019, pp. 56-61. doi: 10.11648/j.sjc.20190703.11

Received: June 1, 2019; Accepted: July 9, 2019; Published: July 22, 2019

\begin{abstract}
A mathematical process of enumeration of Kekule structures of any polybenzenoïd monoradical having only linear assemblies of benzenoid hydrocarbons is given by using a method which consisted of the excision of the radical center $C^{\bullet}$ in order to obtain a conjugated hydrocarbon having a 12 -annulene core ring appended to one or more polybenzenoïd fragments, the partition of the resulting conjugated hydrocarbon into smaller independent resonant circuits. Polyhex monoradical skeletons are obtained from a building up procedure which consists to fuse in different ways the acenaphtyl moiety with one or more polybenzenoïd fragments. Mathematical formulas are established for three cases of assemblies, one edge linear assemblies, two edges linear assemblies with two linear polybenzenoid fragments located at right angles $\theta=60^{\circ}$, $120^{\circ}$ or $180^{\circ}$ and three edges linear assemblies with three linear polybenzenoid fragments located at right angles $\theta=60^{\circ}, 120^{\circ}$ or $180^{\circ}$. Results show that the number of Kekule structures of any polybenzenoïd monoradical having only linear assemblies of benzenoid hydrocarbons increases with the number of fused benzenoids according to results compiled in the table for $h$ equal 1,2,3 and 4. Difficulty to represent graphs when the number of structures increases with the number of benzenoids cannot be neglected.
\end{abstract}

Keywords: Polyhex, Enumeration, Kekule, Monoradicals, Linear Assemblies

\section{Introduction}

Polycyclic aromatic hydrocarbons are the most abundant class of molecules in the known universe, occurring in meteorites, carbon stars in interstellar space and as pyrolytic products in the combustion engine. The discovery and introduction of the concept of resonance embedding in this chemical compounds family has generated the problem of enumerating Kekule structures of polyhex molecules having several conjugated double bonds. Many publications dealing with the problem of enumeration of resonance structures prove that this problem is far from being exhausted. The enumeration of polyhex hydrocarbons dates back to 1964 [1]. From that day, generation and enumeration of polyhexes has attracted the interest of many researchers. Kekule structures in polyhexes, or particular classes of polyhexes such as cata- condensed or pericondensed benzenoids, coronoids or helicenes have been extensively studied [2-15]. Indeed, a whole book by Cyvin and Gutman [14] is devoted to that topic. It is also discussed at length in several surveys of two recent volumes on Advances in the theory of benzenoid hydrocarbons $[16,17]$ and in numerous papers cited there. In this work, we are interested in determining the number of Kekule structures of any polybenzenoïd monoradical having only symmetrical linear assemblies of benzenoid hydrocarbons. To simplify the language, we will speak of assemblies of benzenoid hydrocarbons on the acenaphtyl moiety. Beyond the direct formulas of computation established, this work provides another insight on the problem of enumeration of Kekule structures of polyhexes monoradicals. This paper contribute to the extensive work done on enumerations of any polybenzenoïd monoradical 
which still to be documented comparatively to others category of polyhexes such as fusene or perifusene.

\section{Generation of Polyhex Monoradical Skeletons}

Polyhex monoradical skeletons examined in this work are obtained from a building up procedure which consists to fuse in different ways the acenaphtyl moiety with one or more polybenzenoïd fragments.

Various types of fusion or assembly may occur. The edge to edge fusion which may take place between one edge of $\mathrm{A}$ according to the orientations 1,2,3 or 1',2',3' and one terminal edge of a linear polyacene B. Successive repetition of this process generates a monoradical having a trihex core assembled to two or three linear polybenzenoid fragments located at right angles $\theta=60^{\circ}, 120^{\circ}$ or $180^{\circ}$ (figure 1 ).



Figure 1. Acenaphtyl moiety and orientation of linear fusion.

\section{Enumeration of Kekule Structures of a Polybenzenoïd Monoradical}

\subsection{Mathematical Formulation}

The enumeration of Kekule structures of any polybenzenoïd monoradical utilizes two steps:

(1) the deletion of the radical center $C^{\bullet}$ in order to obtain a conjugated hydrocarbon having a 12 -annulene core ring appended to one or more polybenzenoïd fragments and

(2) the partition of the resulting conjugated hydrocarbon into smaller independent resonant circuits.

The computation as prescribed by the literature [15] of the number of Kekule structures of these independent circuits identified in step two aforementioned.

The determination of the number of Kekule structure of the monoradical using the principle of combination and taking into account the possibly independent resonant circuits.

The deletion of the radical center $C^{\bullet}$ in $\mathrm{A}_{\mathrm{I}}$ and $\mathrm{A}_{\mathrm{II}}$ is depicted on the following diagrams:

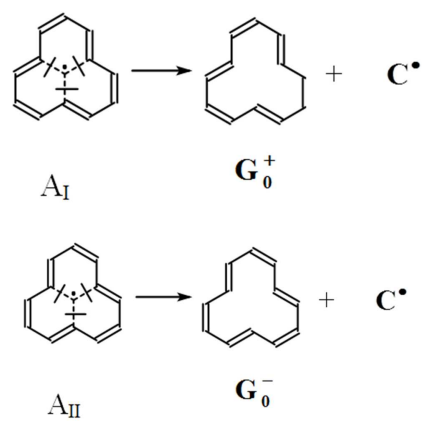

Where the resulting substructures $G_{0}^{+}$and $G_{0}^{-}$are planar 12 -annulene rings containing six double bonds rotating in the clockwise (+sign) and anti clockwise (-sign) directions.

Such a distinction between $G_{0}^{+}$and $G_{0}^{-}$generates the following theorems:

Theorem 1: The number of Kekule structures of the unidirectional circuits $G_{0}^{+}$and $G_{0}^{-}$are respectively:

$$
\mathrm{K}\left(G_{0}^{+}\right)=\mathrm{K}\left(G_{0}^{-}\right)=1
$$

Theorem 2: The number of Kekule structures of a single monoradical center $C^{\bullet}$ which exhibit an invariant electronic structure is $\mathrm{K}\left(C^{\bullet}\right)=1$

If we claim that the two mesomeric forms of the trihex monoradical result from the interactions of $G_{0}^{+}$and $G_{0}^{-}$with $C^{\bullet}$ therefore:

$$
\begin{aligned}
& \mathrm{K}\left(\mathrm{A}_{\mathrm{I}}\right)=\mathrm{K}\left(G_{0}^{+}\right) \times \mathrm{K}\left(C^{\bullet}\right)=1 \\
& \mathrm{~K}\left(\mathrm{~A}_{\mathrm{II}}\right)=\mathrm{K}\left(G_{0}^{-}\right) \times \mathrm{K}\left(C^{\bullet}\right)=1
\end{aligned}
$$

The excision of $\left(C^{\bullet}\right)$ of a monoradical suppresses 3 internal edges and generates a parent conjugated hydrocarbon contains a planar 12 annulene ring possessing a resonant circuit of type $G_{0}^{+}$or $G_{0}^{-}$.

The dissection of the parent conjugated circuits of type $G_{0}^{+}$and $G_{0}^{-}$into smaller independent benzenoïd fragments is operated at a second step, with respect to the following conditions:

a) To position the cutting lines, one may start from the core of the structure and move toward the terminal edges of each polybenzenoïd branch.

b) The orientation of each cutting line follows adjacent hexagonal rings and non-adjacent single bonds inside each hexagonal ring.

c) A cutting dissection line does not bissect a double bond.

d) The benzenoïd fragment to retain during a cutting in any direction is one which has a maximum number of hexagonal rings in resonance.

If the previous conditions are satisfied during the dissection of the parent conjugated circuit one may obtain the solution of the problem of the enumeration of Kekule structures of any monoradical $G_{0}$ by applying the following 
principles.

Now let $G_{0}$ denote any polyhex monoradical having a trihex core appended to one or more polybenzenoïd fragments.

As previously observed the core of $\mathbf{G}_{\mathbf{0}}$ contains two central resonant circuits $G_{0}^{+}$and $G_{0}^{-}$, which may induce two mesomeric resonant forms $G_{0}^{+}$and $G_{0}^{-}$containing a 12annulene ring in clockwise (+sign) and anticlockwise (-sign) rotation respectively.

The mesomeric forms $G_{0}^{+}$and $G_{0}^{-}$generated the following instantaneous equilibria.

$$
G_{0}^{+} \leftrightarrow G_{0} \leftrightarrow G_{0}^{-}
$$

Let $\mathrm{K}\left(G_{0}\right), \mathrm{K}\left(G_{0}^{+}\right)$and $\mathrm{K}\left(G_{0}^{-}\right)$denote the number of kekule structures of $G_{0}, G_{0}^{+}, G_{0}^{-}$. From (4) one way may deduce the following relationship:

$$
\mathrm{K}\left(G_{0}\right)=\mathrm{K}\left(G_{0}^{+}\right)+\mathrm{K}\left(G_{0}^{-}\right)
$$

The excision and dissections processes applied to the resonant forms $G_{0}^{+}$and $G_{0}^{-}$of the polyhex are collected in the following set of dissections or partitions:

$$
\begin{gathered}
G_{0}^{+}=\left\{\mathrm{D}_{01}, \mathrm{D}_{02}, \ldots, \mathrm{D}_{0 \mathrm{i}}, \ldots, \mathrm{D}_{0 \mathrm{j}}, C^{\bullet}, \delta\right\} \\
G_{0}^{-}=\left\{\mathrm{D}_{01}, \mathrm{D}_{02}, \ldots, \mathrm{D}_{0 \mathrm{r}}, \ldots, \mathrm{D}_{0 \mathrm{k}}, C^{\bullet}, \delta^{\prime}\right\}
\end{gathered}
$$

The elements $\mathrm{D}_{0 \mathrm{i}}(1 \leq \mathrm{i} \leq \mathrm{j})$ and $\mathrm{D}_{0 \mathrm{r}}{ }^{\prime}(1 \leq \mathrm{r} \leq \mathrm{k})$ have polybenzenoïd classical structures, $C^{\bullet}$ is the radical center, while $\delta$ and $\delta^{\prime}$ are residual non resonance fragments (possessing one or more non conjugated double bonds)

If $G_{0}^{+}$and $G_{0}^{-}$contain respectively $\mathrm{j}$ and $\mathrm{k}$ independent polybenzenoïd substructures, therefore:

$$
\begin{gathered}
\mathrm{K}\left(G_{0}^{+}\right)=\prod_{i=1}^{j} K\left(D_{0 i}\right) \\
\mathrm{K}\left(G_{0}^{-}\right)=\prod_{r=1}^{k} K\left(D_{0 r}^{\prime}\right) \\
\mathrm{K}\left(G_{0}\right)=\prod_{i=1}^{j} K\left(D_{0 i}\right)+\prod_{r=1}^{k} K\left(D_{0 r}^{\prime}\right)
\end{gathered}
$$

The enumeration of Kekule structures for numerous conjugated hydrocarbons is intensively documented in chemical literature. [15]. However, we recalled that the number of Kékulé structures of linear polybenzenoids $\mathrm{G}_{\mathrm{i}}$ is

$$
\mathrm{K}\left(\mathrm{G}_{\mathrm{i}}\right)=\mathrm{h}_{\mathrm{i}}+1
$$

Where $h_{i}$ is the number of benzenoids.

In equation (1)-(15), $\mathrm{K}\left(\mathrm{D}_{0 \mathrm{i}}\right)$ and $\mathrm{K}\left(\mathrm{D}^{\prime}{ }^{\prime}{ }_{\mathrm{r}}\right)$ are the number of Kekule structures of the polybenzenoïd with one or many dissections $\mathrm{D}_{0 \mathrm{i}}$ and $\mathrm{D}^{\prime}{ }_{0 \mathrm{r}}$ respectively.

We have applied the partition processes and (9)-(15) to the enumeration of Kekule structures for the following series of polyhex monoradicals having linear assemblies.

Case 1: One edge linear assemblies

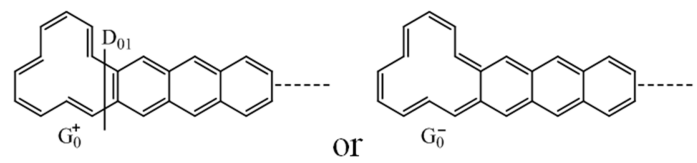

We have one dissection $\mathrm{D}_{01}$ on $G_{0}^{+}$

$$
\begin{gathered}
\mathrm{K}\left(\mathrm{G}_{0}\right)=\mathrm{K}\left(\mathrm{D}_{01}\right)+1 \\
\mathrm{~K}\left(\mathrm{G}_{0}\right)=\mathrm{h}_{1}+1+1=\mathrm{h}_{1}+2
\end{gathered}
$$

Case 2: Two edges linear assemblies

We will distinguish here the various possible assemblies. These assemblies can be at $60^{\circ}, 120^{\circ}, 180^{\circ}$ from each other.

For assemblies of $60^{\circ}$ :
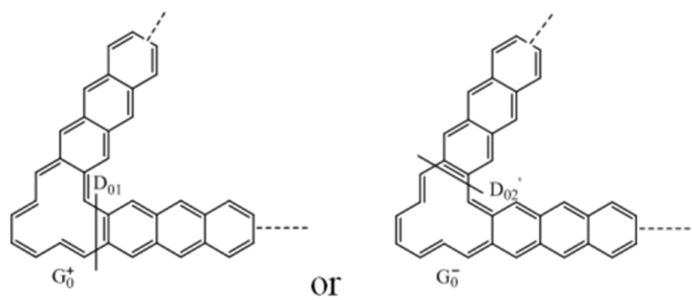

$$
\mathrm{K}\left(\mathrm{G}_{0}\right)=\mathrm{K}\left(\mathrm{D}_{01}\right)+\mathrm{K}\left(\mathrm{D}_{02}{ }^{\prime}\right)
$$

$$
\mathrm{K}\left(\mathrm{G}_{0}\right)=\mathrm{h}_{1}+1+\mathrm{h}_{2}+1=\mathrm{h}_{1}+\mathrm{h}_{2}+2
$$

For assemblies of $180^{\circ}$ :
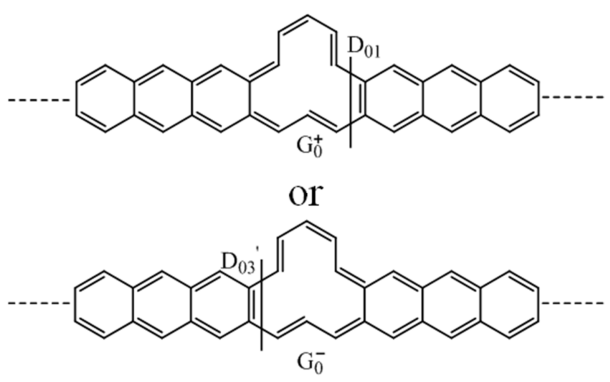

$$
\begin{gathered}
\mathrm{K}\left(\mathrm{G}_{0}\right)=\mathrm{K}\left(\mathrm{D}_{01}\right)+\mathrm{K}\left(\mathrm{D}_{03}{ }^{\prime}\right) \\
\mathrm{K}\left(\mathrm{G}_{0}\right)=\mathrm{h}_{1}+1+\mathrm{h}_{3}+1=\mathrm{h}_{1}+\mathrm{h}_{3}+2
\end{gathered}
$$

For an assemblies of $120^{\circ}$ :




59

Alphonse Emadak et al:: Enumeration of Kekule Structures of Polyhex Monoradical Having

Only Linear Assemblies of Benzenoid Hydrocarbons

Case 3: Three edges linear assemblies

The three linear branches can be positioned in two different ways on the various assembly sites offered by the perinaphthyl.

First way:
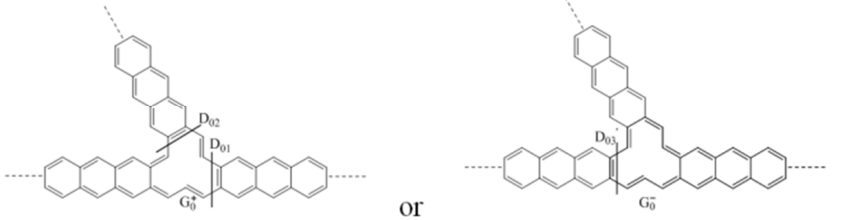

$$
\begin{gathered}
\mathrm{K}\left(\mathrm{G}_{0}\right)=\mathrm{K}\left(\mathrm{D}_{01}\right) \mathrm{xK}\left(\mathrm{D}_{02}\right)+\mathrm{K}\left(\mathrm{D}_{03}{ }^{\prime}\right) \\
\mathrm{K}\left(\mathrm{G}_{0}\right)=\left(\mathrm{h}_{1}+1\right)\left(\mathrm{h}_{2}+1\right)+\left(\mathrm{h}_{3}+1\right)
\end{gathered}
$$

Second way:

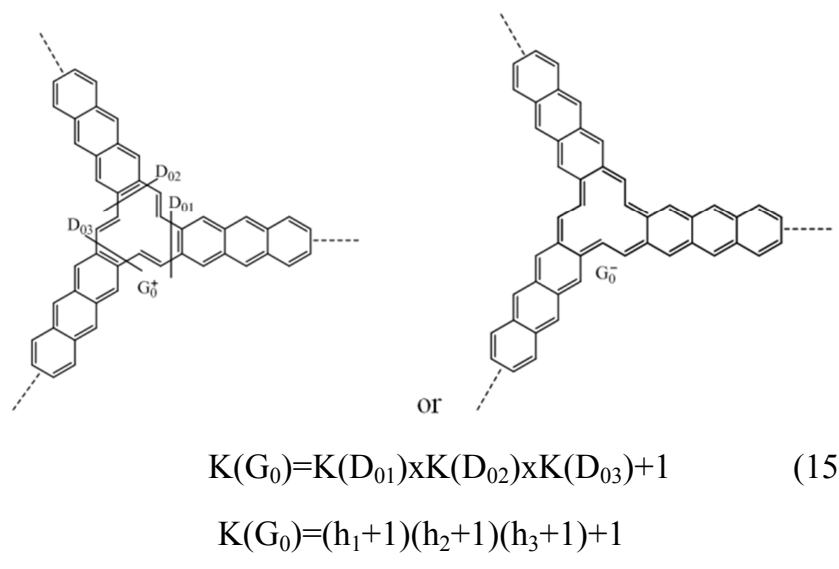

3.2. Application, Results and Graphical Representations

The application is focusing on the enumeration of Kekule structures of any polybenzenoïd monoradical having only symmetrical linear assemblies of benzenoid hydrocarbons. Therefore $h_{i}=h$. The results of computation obtained is followed by graphical representations of Kekule structures.

Case 1: One edge linear assemblies

Series of a single linear assembly are enumerate using (10) where $h_{i}=h$ and $\mathrm{K}\left(\mathrm{G}_{0}\right)=h_{l}+1+1=h_{l}+2=h+2$

For $h=0, \mathrm{~K}\left(\mathrm{G}_{0}\right)=2$<smiles></smiles><smiles></smiles>

For $h=1, \mathrm{~K}\left(\mathrm{G}_{0}\right)=3$<smiles></smiles><smiles>c1ccc2cc3ccccc3cc2c1</smiles><smiles></smiles>

For $h=2, \mathrm{~K}\left(\mathrm{G}_{0}\right)=4$



Case 2: Two edges linear assemblies

Series of a two linear assemblies are enumerate using (11), (12) and (13) where $h_{i}=h$.

For $60^{\circ}$ assemblies, we have

$$
\mathrm{K}\left(\mathrm{G}_{0}\right)=\mathrm{h}_{1}+1+\mathrm{h}_{2}+1=\mathrm{h}_{1}+\mathrm{h}_{2}+2=2(\mathrm{~h}+1)
$$

For $h=1, \mathrm{~K}\left(\mathrm{G}_{0}\right)=4$

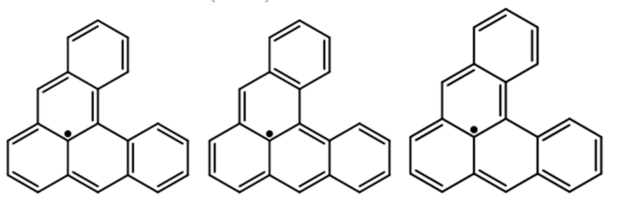<smiles>C1=CC=C2C(=c3ccccc3=Cc3ccccc32)C=C1</smiles>

For $h=1, \mathrm{~K}\left(\mathrm{G}_{0}\right)=6$

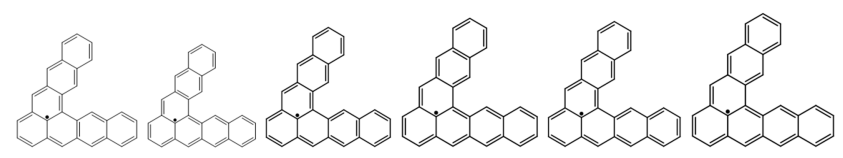

For $180^{\circ}$ assemblies, we have

$$
\mathrm{K}\left(\mathrm{G}_{0}\right)=\mathrm{K}\left(\mathrm{G}_{0}\right)=\mathrm{h}_{1}+1+\mathrm{h}_{3}+1=\mathrm{h}_{1}+\mathrm{h}_{3}+2=2(\mathrm{~h}+1)
$$

For $h=1, \mathrm{~K}\left(\mathrm{G}_{0}\right)=4$

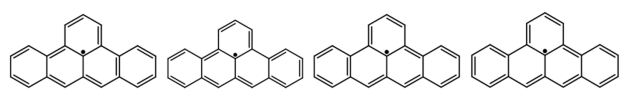

For $h=1, \mathrm{~K}\left(\mathrm{G}_{0}\right)=6$

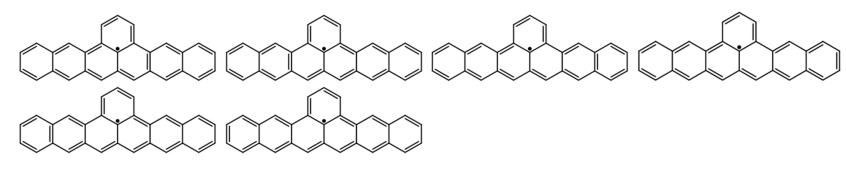

For $120^{\circ}$ assemblies, we have

$$
\mathrm{K}\left(\mathrm{G}_{0}\right)=\left(\mathrm{h}_{1}+1\right)\left(\mathrm{h}_{2}+1\right)+1=(\mathrm{h}+1)^{2}+1
$$

For $h=1, \mathrm{~K}\left(\mathrm{G}_{0}\right)=5$

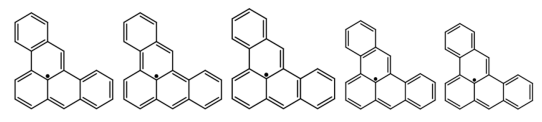

For $h=2, \mathrm{~K}\left(\mathrm{G}_{0}\right)=10$

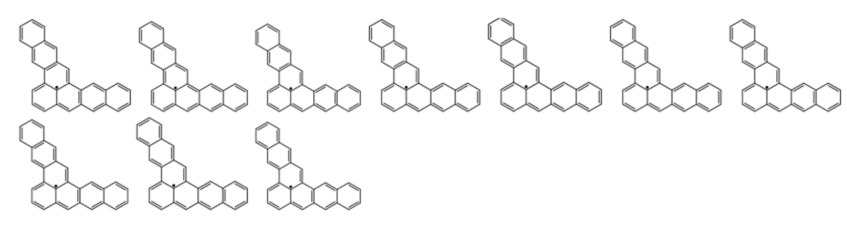

Case 3: Three edges linear assemblies

Series of a three linear assemblies are enumerate using (14) and (15) where $h_{i}=h$.

For the first type of assemblies, we have

$$
\mathrm{K}\left(\mathrm{G}_{0}\right)=\left(\mathrm{h}_{1}+1\right)\left(\mathrm{h}_{2}+1\right)+\left(\mathrm{h}_{3}+1\right)=(\mathrm{h}+1)(\mathrm{h}+2)
$$

For $h=1, \mathrm{~K}\left(\mathrm{G}_{0}\right)=6$ 
Science Journal of Chemistry 2019; 7(3): 56-61

60

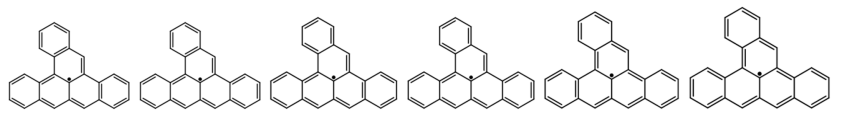

For $h=2, \mathrm{~K}\left(\mathrm{G}_{0}\right)=12$

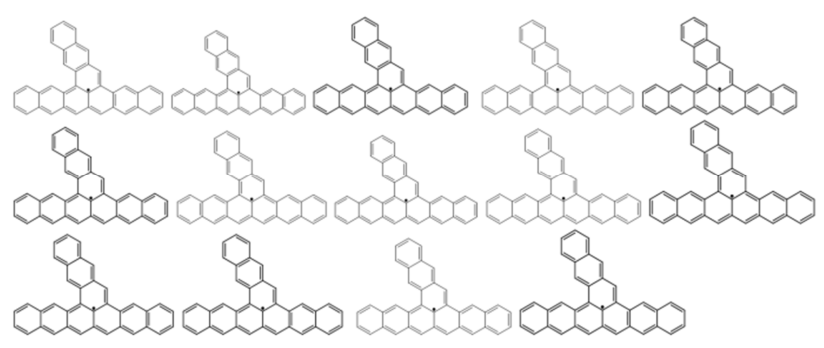

For the second type of assemblies, we have

$$
\mathrm{K}\left(\mathrm{G}_{0}\right)=\left(\mathrm{h}_{1}+1\right)\left(\mathrm{h}_{2}+1\right)\left(\mathrm{h}_{3}+1\right)+1=(\mathrm{h}+1)^{3}+1
$$

For $h=1, \mathrm{~K}\left(\mathrm{G}_{0}\right)=9$

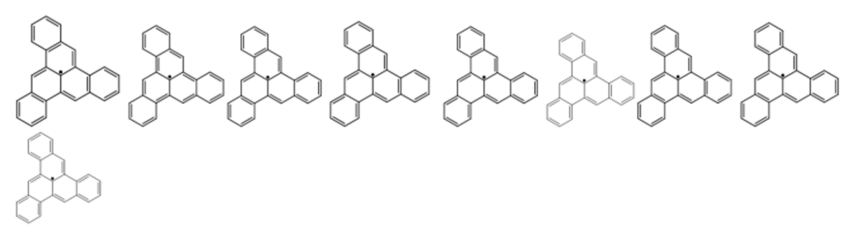

For $h=2, \mathrm{~K}\left(\mathrm{G}_{0}\right)=28$

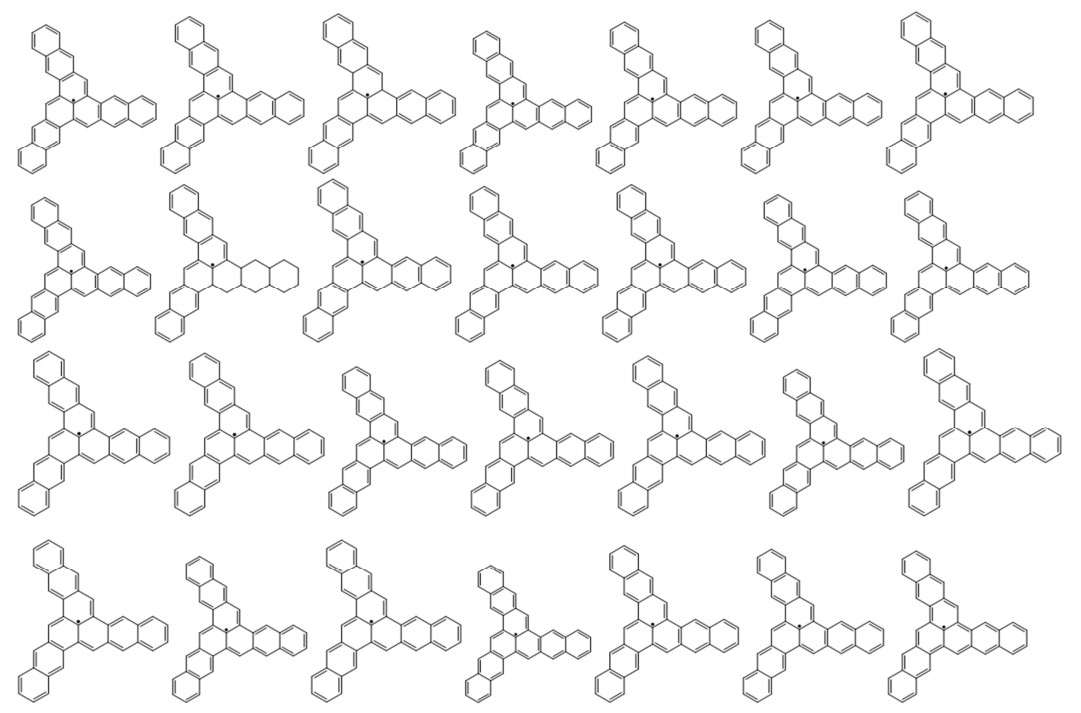

Table 1. Results summary of the enumeration polyhex monoradical with linear assembled polybenzenoïds.

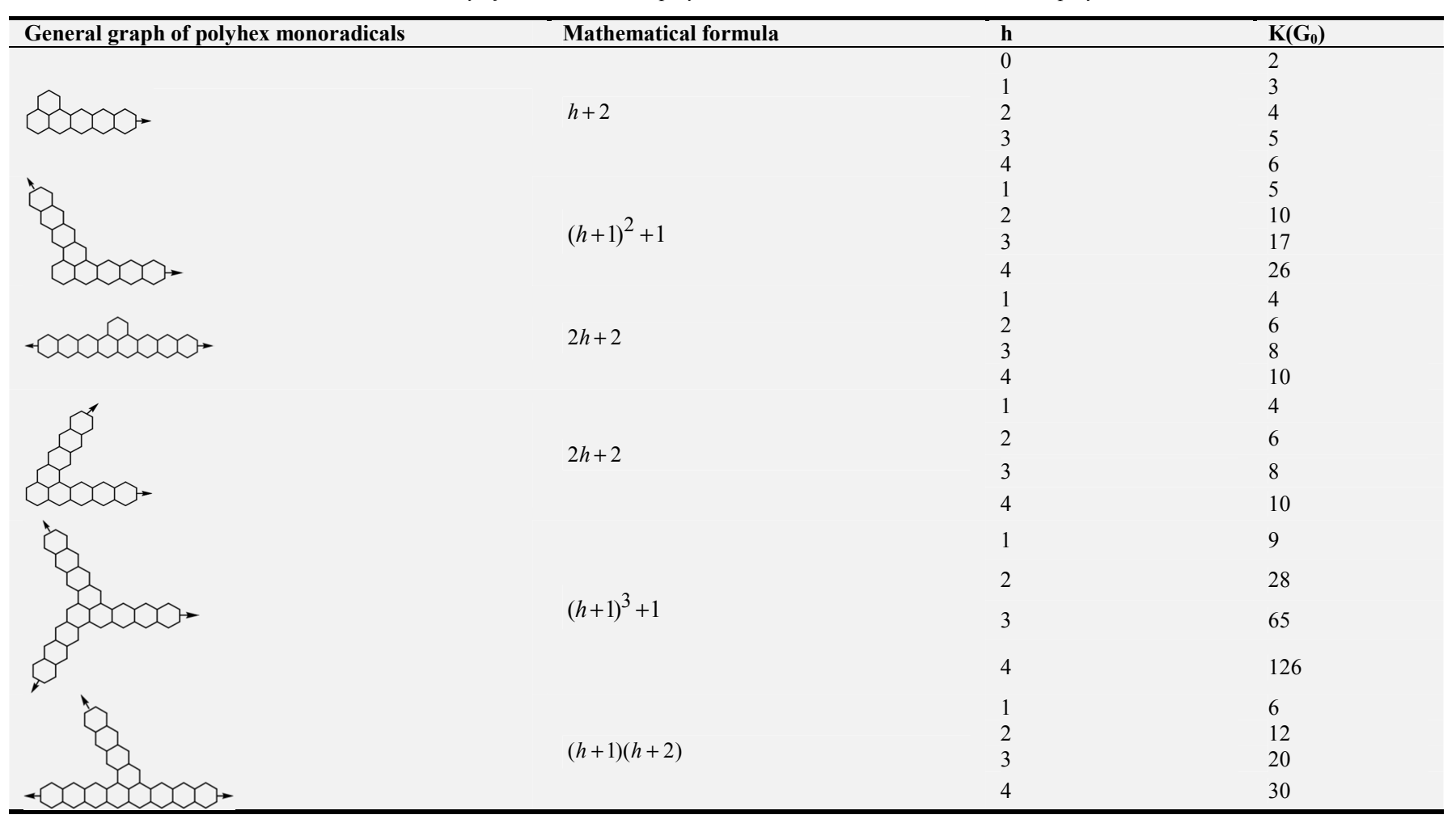


According to the above table of results, the mathematical formulas of the number of kekule structures of polyhexes monoradical having identical two edges linear polybenzenoids are invariant when the two linear polybenzenoid fragments are located at angles $\theta=60^{\circ}$ or $180^{\circ}$. For $\mathrm{h}=3$ and above, it is observed more than $50 \%$ gap between numbers kekule of the two cases of structures of polyhexes monoradical having identical three edges linear polybenzenoids with different angles. In general, the number of kekule structures increases with $\mathrm{h}$ for any cases.

\section{Conclusion}

The results summarized in this paper are expected to eventually lead to a better understanding of how compute the number of kekule structures of polyhexes monoradical having only identical linear polybenzenoids. We did not carry out the computation of cases where $h_{1} \neq h_{2} \neq \ldots \neq h_{i}$ because formulas derived can be used to obtain the number of those monoradical polycyclic aromatic hydrocarbons. However, this case is relevant to be studied in future papers with the involvement of combinatorial methodology combined with group theory approach. We noticed the difficulty to represent graphs when the number of structures increases with the number of benzenoids. This complexity challenges us to develop easy and appropriate methods that can generate all the structures without duplication whatever the size of the graphs.

\section{Acknowledgements}

This research was supported by the Ministry of Higher Education of the Republic of Cameroon through the modernization research allowances. Authors also thank the University of Yaounde' I and High Teacher Training College (Cameroon) for the use of their infrastructural facilities.

\section{References}

[1] R. Tosic, D. Masulovic, I. Stojmenovic, J. Brunvoll, B. N. Cyvin and S. J. Cyvin, "Enumeration of Polyhex Hydrocarbons to $\mathrm{h}=17$," J. Chem. Inf. Comput. Sci., vol. 35, pp. 181-187, 1995.

[2] N. Trinajstic, S. Nikolic, J. V. Knop, W. R. Müller, and K. Szymanski, Computational Chemical Graph Theory, Ellis Horwood, New York, London 1991.

[3] N. Trinajstic, Chemical Graph Theory (2nd ed.), CRC, Boca Raton, London, Tokyo 1992.
[4] I. Gutman and S. J. Cyvin, Introduction to the Theory of Benzenoid Hydrocarbons, Springer, Berlin 1989.

[5] I. Gutman and J. Cioslowski, Publ. Inst. Math. Beograd 42, 21 (1987).

[6] I. Gutman, Match 13, 173 (1982).

[7] S. J. Cyvin and I. Gutman, Z. Naturforsch. 41a, 1079 (1986).

[8] B. N. Cyvin, J. Brunvoll, S. J. Cyvin, and I. Gutman, Match 21, 301 (1986).

[9] S. J. Cyvin, J. Brunvoll, and B. N. Cyvin, Z. Naturforsch. 41a, 1429 (1986).

[10] S. J. Cyvin, Match 20, 165 (1986).

[11] R. Chen and S. J. Cyvin, Match 22, 175 (1987).

[12] A. T. Balaban, J. Brun voll, B. N. Cyvin, and S. J. Cyvin, Tetrahedron 44, 221 (1988).

[13] F. Zhang, R. Chen, and X. Guo, Graphs Comb. 1, 383 (1985).

[14] A. V. Kostochka, Proc. 30. Int. Wiss. Koll. TH Ilmenau F, 49 (1985).

[15] S. J. Cyvin and I. Gutman, Kekule Structures in Benzenoid Hydrocarbons (Lecture Notes in Chemistry 46), Springer, Berlin 1988.

[16] I. Gutman and S. J. Cyvin (eds.), Topics Curr. Chem. (Advances in the Theory of Benzenoid Hydrocarbons) 153 (1990).

[17] I. Gutman (ed.), Topics Curr. Chem. (Advances in the Theory of Benzenoid Hydrocarbons II) 162 (1992).

[18] P. John, Z. Phys. Chem. 271, 941 (1990).

[19] A. T. Balaban, J. Brunvoll, B. N. Cyvin, and S. J. Cyvin, Tetrahedron 44, 221 (1988).

[20] R. Bellman and S. Dreyfus, Applied Dynamic Programming, Princeton Press, Princeton, New Jersey 1962.

[21] H. Hosoya, Topics Curr. Chem. 153, 273 (1990).

[22] I. Gutman, J. Serb. Chem. Soc. 11, 607 (1988).

[23] A. T. Balaban, Pure and Appl. Chem. 65, 1 (1993).

[24] A. T. Balaban, X. Liu, S. J. Cyvin, and D. J. Klein, Benzenoids with Maximum Kekule Structure Counts for Given Number of Hexagons, J. Chem. Inf. Comput. Sei. 33, 429 (1993).

[25] R. Bellman, Dynamic Programming, Princeton Press, Princeton, New Jersey 1957. 